\title{
MODELO DE SISTEMA DA INFORMAÇÃO PARA GERAR FEEDBACK NO ENSINO BASEADO NA TEORIA DA ATIVIDADE
}

\author{
MODELO DE SISTEMA DE INFORMACIÓN PARA \\ GENERAR FEEDBACK EN LA ENSEÑANZA BASADO \\ EN LA TEORÍA DE LA ACTIVIDAD
}

\author{
Emilio Evaristo de Sousaa \\ Cláudio Gottschalg Duque ${ }^{b}$
}

\begin{abstract}
RESUMO
Introdução: O feedback é a informação sobre a performance de uma pessoa em uma determinada tarefa. A falta desse item na sala de aula pode trazer diversos prejuízos para a qualidade do ensino. Objetivo: $O$ estudo busca analisar a percepção do aluno para gerar informação relevante ao professor em forma de feedback. Metodologia: Para tanto, propomos uma modelagem inovadora com aspectos da teoria da atividade, multimodalidade e uso de ontologia. Resultados: Como resultado, pretende-se criar uma ontologia das salas de aulas que determine as atividades do professor que podem ser auxiliadas com a percepção dos alunos na educação. Conclusões: A possibilidade da aplicação de seus resultados no auxílio e capacitação dos professores, provendo um meio para o uso da informação no enfrentamento dos desafios da sala de aula e qualificação do ensino.
\end{abstract}

Descritores: Sistemas de Informação. Feedback. Educação. Teoria da Atividade. Multimodalidade. Ontologia.

\footnotetext{
a Doutor em Ciência da Informação pela Universidade de Brasília. Professor na Secretaria de Educação do Distrito Federal. E-mail:emilioevaristo@hotmail.com

b Doutor em Produção e Gestão da Informação pela Escola de Ciência da Informação. Professor Adjunto da Faculdade de Ciência da Informação da Universidade de Brasília (FClUnB). E-mail: klaussherzog@gmail.com
} 


\section{INTRODUÇÃO}

Nesta pesquisa procuramos entender um pouco mais sobre o feedback e seu impacto no processo de aprendizagem. Rinvolucri (1994) defende que o termo feedback teve sua origem na biologia e refere-se à mensagem que retorna a um organismo após sua ação no ambiente. Na edição virtual do dicionário inglês Oxford (2015), o feedback é uma informação sobre reações a um produto ou a performance de uma pessoa numa tarefa, a qual é usada como base para sua melhora. No contexto educacional, Penny Ur (1996) define feedback como uma informação que é dada ao aprendiz sobre seu desempenho em uma tarefa de aprendizagem, geralmente com o objetivo de melhorar seu desempenho. Assim, como podemos inferir pelas definições apresentadas, o conceito de feedback está relacionado com informação, ação e desempenho.

Por diversos meios de comunicação, a informação emitida "viaja" ao receptor com o intuito de oferecer uma informação que determine uma melhora em uma ação específica, criando uma complexa arquitetura. Para o desenvolvimento de uma arquitetura da informação/feedback que tenha como consequência uma melhora no desempenho, alguns aspectos devem ser considerados como: Qual a melhor forma de prover essa informação? Quais canais de informação devem ser considerados? Devemos fornecer feedback para quais ações? Como oferecer um feedback relevante? No caso do ambiente educacional, os papéis de professor e aluno devem ser considerados e estudados de forma aprofundada, tornando um desafio ainda maior uma proposta de arquitetura para auxílio no processo de aprendizagem.

No campo do ensino, feedback constitui um elemento para o exercício das competências de ensino. Ele contribui para a detecção de características positivas da interferência no ensino, bem como a detecção dos pontos fracos de modo a melhorá-los (FRAGOULIS; DIAMANTAKI, 2012). Diversos estudos apontam para o feedback como um elemento essencial que permite melhorar e desenvolver a aprendizagem dos alunos (NICOL; MACFARLANE-DICK, 2006; LIZZIO; WILSON, 2008; TANG; HARRISON, 2011; ROBINSON et al., 2013; 
ORSMOND et al., 2013), apesar de no ensino brasileiro não terem sido encontrados relatos de pesquisa relacionados ao tema.

Entretanto, o enfoque das pesquisas sobre feedback não limita-se somente ao impacto nos alunos. O recente estudo (MET, 2014), apoiado pela fundação Bill e Melinda Gates, aponta que os professores são mais importantes para o aprendizado do aluno do que qualquer outro fator na escola. Diante disso, concentraram-se nos estudos de feedback aos professores por meio dos pares, concluindo que o feedback auxiliou professores a aumentarem a qualidade do ensino, sendo essa melhora fundamental para o sucesso do aluno. Em outra pesquisa, foi realizado estudo para identificar o impacto do feedback no processo de formação do docente. A conclusão foi que a falta de feedback implicou em uma não melhoria do progresso ou das competências dos formandos em desenvolvimento (LERNER, 2002). Apesar desse maior interesse em fornecer feedback aos professores, as pesquisas e práticas adotadas ainda são imaturas e aplicadas em pequenas iniciativas. Além disso, essas pesquisas ainda possuem um alto custo por necessitarem de mentores, tutores ou dos pares para que o feedback aconteça.

Para atingir uma aprendizagem efetiva, a Sociedade do Conhecimento exige a reorganização dos ambientes de aprendizagem, a compreensão das relações de espaço-temporalidade e mudanças nas relações de ensino e de aprendizagem, porquanto, a conexão da escola à Sociedade da Informação implica assegurar a sua pertinência, enquanto instituição responsável pela formação de sujeitos capazes de atuação na sociedade tecnológica (SANTOS, 2011).

Diante de tantos desafios e abordagens, percebe-se a dificuldade para mensurar as melhores práticas nos ambientes de aprendizagem. Em ambientes educacionais presenciais, professores e alunos estão em constante interação e contam com elementos verbais e não-verbais. Em uma abordagem sobre o assunto, Paiva (2003) descreve que em tais ambientes os alunos observam a atuação do professor e percebem seus movimentos, tais como, expressões faciais, gestos, acenos com a cabeça etc. $O$ feedback refere-se a várias mensagens (visual, áudio, verbal, não-verbal), que são recebidas pelos 
professores sobre os resultados das ações de ensino (FRAGOULIS; DIAMANTAKI, 2012). Assim, cada artefato da prática do professor - aonde avaliações do estudante sobre a eficácia de um professor, observações de sala de aula diretas, ou as melhoras anteriores ou recentes dos estudantes - são potencialmente úteis para identificar os pontos fortes e fracos do professor e servir de informação para traçar planos para alcançar o sucesso com estudantes futuros. Todos esses dados podem, por meio de um tratamento, servir de informação para aperfeiçoar o desenvolvimento profissional dos professores. Por que essa informação proveniente dos alunos não são utilizadas nos ambientes educacionais? Pouco se sabe sobre a utilização desses dados para melhora profissional do professor, do processo de ensinoaprendizagem ou a organização dessa informação para fornecer um feedback relevante nos ambientes educacionais.

Um dos desafios na organização da informação para promover feedback em ambientes educacionais, no entanto, é a modelagem da relação entre os dados dentro da sala de aula, seu contexto e a relevância para as partes envolvidas. De uma perspectiva da arquitetura da informação, isto significa definir quais dados e a forma que deverão ser capturados, baseado em quais relações de aprendizagem são relevantes em sala de aula, organizá-los e traduzir para o alto nível de conceitos ou termos que seria intuitivo para o professor fazer uso. Para definir quais dados e forma que deverão ser capturados, a abordagem da multimodalidade fornece ferramentas úteis para auxiliar nos diversos canais de informação que navega o feedback. A percepção dos alunos pode ser um papel central na criação de um modelo de geração de feedback relevante ao professor. Antunes (2008) ressalta a importância da ajuda do mediador no conceito de aprendizagem defendido por Vygotsky, porém evidencia a necessidade de associar os saberes que leva aos esquemas de conhecimento que os alunos possuem, posto que são estes os geradores das significações, essenciais em uma aprendizagem consciente.

Avançar para um modelo que elabore feedback relevante aos professores requer a análise da percepção das atividades docentes e a definição das estruturas informacionais nos ambientes educacionais. O objetivo 
geral dessa pesquisa é identificar as atividades de relevância da percepção dos estudantes na geração de feedbacks para professores em ambientes educacionais, e espera-se alcançar este objetivo por meio de uma ontologia das atividades multimodais da sala de aula.

\section{FUNDAMENTAÇÃO TEÓRICA}

Nesta seção são apresentados os conceitos teóricos sobre teoria da atividade, multimodalidade e ontologia, estabelecendo uma base para a verificação proposta neste trabalho.

\subsection{TeOria da ATIVIDADE}

A Teoria da Atividade teve sua origem na psicologia da União Soviética, como uma alternativa Marxista à ortodoxa psicologia comportamental do ocidente (WILSON, 2008). Foi proposta como uma teoria humana da consciência e explicação da natureza do comportamento humano. $O$ comportamento humano consiste de atividades tipificadas, e a base proposta pela teoria da atividade é que a consciência é formada por meio da atividade. Devido às traduções do termo original russo deyatel'nost para o inglês activity, um pouco do sentido original se perdeu. De acordo com Kuutti (1996), a teoria da atividade conota o estudo do modo como o comportamento humano age sobre os objetos para transformá-los. Na formulação original de Vygotsky (1978), os artefatos mediadores são substituídos por ferramentas psicológicas, o que significava linguagem, escrita, matemática, mapas e outras estruturas de símbolos.

Engeström (1987) adicionou à idéia de Vygotsky os conceitos de regras e normas, comunidade e divisão do trabalho. As regras e normas constituem o formal e informal, legal, e os limites tradicionais da atividade específica a ser feita. A teoria da atividade aparece na área de educação principalmente por meio dos trabalhos de Vygotsky. A zona de desenvolvimento proximal (VYGOTSKY, 1978) descreve uma zona no processo de aprendizagem onde 
uma ajuda de um par de nível mais avançado pode fazer outro par avançar de nível em um determinado conhecimento. Diante dessa pesquisa, Tolman (1999) analisa que o trabalho de Vygotsky infere sobre a diferença entre o que pode ser conseguido individualmente no cotidiano e o que pode ser conquistado por meio de ações coletivas.

Com as recentes tecnologias, os sistemas de informação podem exercer uma função importante no processo de ensino-aprendizagem. $O$ trabalho cooperativo (professor/aluno ou aluno/aluno) nos ambientes educacionais tem a sua importância destacada pelas recentes pesquisas educacionais. Para que os sistemas de informação possam ser usados de forma efetiva, os usuários devem desenvolver um entendimento compartilhado dos artefatos disponíveis para auxiliar na conclusão de suas tarefas. No campo interdisciplinar da teoria da atividade com educação e teoria da aprendizagem, esses relacionamentos cooperativos ocorridos nos ambientes educacionais são significativos objetos de estudo.

$\mathrm{Na}$ arquitetura dos sistemas de informação atuais existem vários problemas e conflitos para desenvolver um método de sucesso. As tecnologias estão se tornando cada vez mais mutáveis (computadores, smartphones, tablets, smartwatch), e as técnicas e ferramentas utilizadas estão se mostrando insuficientes para os desafios de desenvolver uma arquitetura para esse tipo de material. Além disso, a cada dia cresce a importância do usuário e da sua influência no desenvolvimento das tecnologias. Uma abordagem da teoria da atividade no campo da organização da informação pode apresentar ganhos na construção de modelos informacionais. O foco nos sujeitos, objetos e artefatos mediadores trazem orientação para a aplicação das demais metodologias relacionadas na pesquisa. Portanto, a teoria da atividade nos oferece uma outra lente para estudar os ambientes educacionais e os usuários nesta pesquisa. 


\subsection{ONTOLOGIAS}

O termo "Ontologia" é utilizado em diferentes sentidos nas diferentes áreas do conhecimento. Na disciplina de filosofia, o termo "Ontologia" foi originalmente criado por Aristóteles na área da Metafísica e trata do "Ser enquanto Ser" (VIEIRA, 1995). Ontologia é descrita como o estudo dos atributos que pertencem às coisas devido a sua própria natureza. $\mathrm{Na}$ área da Ciência da Computação, a ontologia refere-se a um tipo especial de objeto da informação ou artefato computacional (GUARINO et al, 2009). Dado um certo domínio do conhecimento humano, todo e qualquer conceito pertencente a este domínio, bem como suas relações e funções são uma ontologia. Alguns autores defendem que ontologia é uma descrição formal e explícita de uma conceitualização compartilhada (GRUBER, 1996). Já outros acreditam que é uma teoria lógica que fornece um relato explícito e parcial de uma conceitualização (GUARINO; GIARETTA, 1995). Essa forma de organizar o conhecimento visa descrever estruturas conceituais de domínios específicos, tornar viável o uso do vocabulário compartilhado de uma maneira coerente e consistente.

Feitosa (2005), afirma que

Desde o início dos anos 90, as ontologias tornaram-se um tópico de pesquisa popular nas áreas de inteligência artificial, engenharia do conhecimento, processamento da linguagem natural e representação do conhecimento. A razão de tal popularidade é acreditar-se que, por seu intermédio, um entendimento comum e compartilhado sobre um determinado domínio de conhecimento poderá ser comunicado tanto entre pessoas como entre computadores (FEITOSA, 2005, p.209).

Uma das características principais das ontologias é que elas permitem compartilhar informações de um domínio, podendo ser utilizada por diversas aplicações. Um requisito para compartilhamento é a padronização da linguagem de representação. Nesse sentido, existem diversas linguagens que foram surgindo para a representação de ontologias que serão apresentadas (CORCHO: GÓMEZ-PÉREZ, 2000).

Em 2001, o W3C criou um grupo de trabalho chamado Web-Ontology 
para definir uma nova linguagem de ontologias para a web semântica. A Web Ontology Language, OWL (SMITH et al, 2004), é uma recomendação apresentada pela W3C como uma linguagem de ontologias para a web. Uma parte das características da DAML+OIL foi utilizada como entrada principal para o desenvolvimento da primeira especificação da linguagem OWL (DEAN et al, 2002). OWL aumenta a expressividade da linguagem, pois permite identificar restrições de propriedade, de equivalência, e quantificadores.

\subsection{MULTIMODALIDADE}

A multimodalidade aborda a representação, comunicação e interação como algo além da língua. Para Kress e Van Leeuwen (2001), a multimodalidade é o uso de vários recursos semióticos na criação de um produto ou evento semiótico. A multimodalidade traz uma abordagem de interpretação além do social, estudando os modos de representação e comunicação e o uso dos recursos semióticos (discurso, escrita, gestos, imagens, postura, olhar) e suas relações dentro de uma cultura.

A linguagem verbal é parte de um conjunto multimodal e o modo mais significativo de comunicação no contexto da aprendizagem e do ensino. Entretanto, a multimodalidade parte do pressuposto que a representação e a comunicação são feitas de múltiplos modos, os quais tem potencial para contribuir igualmente para geração de significado. Royce (2002) e Kress e Van Leeuwen $(1996,2001)$ defendem o ponto de vista de que mesmo a parte puramente lingüística de um texto deve ser interpretada levando-se em conta o papel desempenhado por cada elemento envolvido na cooperação multimodal.

Frente à reconceituação de letramento e pedagogia do letramento, fazse imperativo o desenvolvimento de pesquisas tendo em vista incrementar a natureza multimodal dos textos em papel e meios digitais. Neste desenvolvimento muitos veem como central a relação entre imagem-texto. Poucos trabalhos tem sido direcionados às relações semânticas intersemióticas entre imagens e linguagem para mostrar como os modos visual e verbal, incluindo textos escritos, interagem para construir significados integrados de 
textos multimodais. O desenvolvimento de um sistema semiótico descrevendo a semântica da articulação recíproca de imagem e linguagem ainda está na sua infância. Como imagens e linguagem interagem na construção de significado dentro da perspectiva multimodal, o avanço deste entendimento parece ser crucial na reconceituação de letramento e pedagogia do letramento.

Segue-se, então, que para entender melhor a aprendizagem e ensino no ambiente multimodal da sala de aula contemporânea, é essencial explorar as maneiras pelas quais a informação é representada em todos os modos na sala de aula. O foco aqui, então, é sobre a multimodalidade e suas representações e as potencialidades de aprendizagem nos materiais didáticos, e as formas em que professores e alunos utilizam estes modos através de sua interação na sala de aula.

Nas salas de aulas, mesmo que inconsciente, o valor do emprego dos diferentes modos nas atividades de aprendizagem é abertamente notável (PASTORE, 2003), onde podemos notar uma classificação de atividades de aprendizagem que partem das experiências concretas e se tornam cada vez mais abstratas. A teoria de Dale (1969), segundo a qual os alunos retêm melhor a informação quando fazem, em relação a quando ouvem, lêem ou observam, deu origem a práticas interativas de sala de aula, como simulações ou roleplay, que mais se aproximam da experiência concreta (DIAS, 2011).

Como Kress (2003) observou, "o mundo da comunicação não está parado", e para os professores, isso tem profundas implicações na era digital dentro da sala de aula. Afinal, se o mundo da comunicação não está parado, por que as nossas práticas pedagógicas deveriam estar?

\section{MÉTODOS}

De acordo com Le Coadic (2004), a ciência da informação vale-se de métodos elaborados em outras disciplinas, como a sociologia, a psicologia e os métodos de pesquisa por meio de questionários, entrevistas, observação, etc. Este tópico descreve e demonstra como realizou a abordagem analítica para os 
estudos aplicáveis à sala de aula. Na sala de aula, buscamos entender as atividades desenvolvidas pelo professor, com foco na aprendizagem.

Diante da necessidade da análise do cenário para construção de uma arquitetura da informação facilitadora, os métodos utilizados baseiam-se na teoria da semiótica social da construção do significado (KRESS; VAN LEEUWEN, 2001; MACHIN, 2007; NORRIS, 2004) e na teoria da atividade proposta por Vygotsky (1978) e Leont'ev (1978), a fim de prover subsídios para uma análise dessa complexa relação entre a semiótica social e o processo da aprendizagem em sala de aula.

A utilização da abordagem multimodal evidencia a complexidade das interações e necessidades pedagógicas. Assim, procuramos descrever os recursos disponíveis, no ambiente do ensino presencial, que possam contribuir no processo de ensino-aprendizagem para melhor compreensão. Os professores quando falam, utilizam de outros recursos semióticos para construção do significado, criando um conjunto de configurações. Professores usam gestos enquanto discursam ou chamam atenção com as mãos para determinados pontos no quadro, e ultimamente interagem com computadores e softwares de apresentação na sala de aula.

Diante de tantos recursos utilizados no processo de ensino, construímos uma ontologia, e que nos permitirá entender e facilitar o processo de feedback aos professores. Inicialmente, realizamos uma análise teórica da sala de aula do ensino brasileiro. Para essa análise, efetivamos o primeiro processo dentro do contexto construtivista e multimodal da sala de aula, com uma perspectiva teórica sobre comunicação e, mais especificamente no ensino e aprendizagem como uma realização multimodal.

A ontologia foi construída por meio dos sete passos sugeridos no método 101 (NOY; MCGUINNESS, 2001). A linguagem que utilizamos na construção da ontologia foi a OWL - Ontology Web Language, padrão recomendado pela W3C. A ferramenta para construção da ontologia foi o Protegè na versão 4.1. Demonstramos em 4 super-classes e suas demais subclasses os resultados da pesquisa e a visão construtivista multimodal para o ambiente da sala de aula, objetivando fornecer (posteriormente) feedback 
que seja relevante para o auxílio da prática docente. Essas atividades foram decompostas detalhadamente para poder determinar a percepção dos estudantes acerca de cada uma no futuro. Assim, o requisito ideal de oferecer um feedback detalhado e objetivo, conforme proposto por Williams (2005), poderá ser atingido.

\section{RESULTADOS}

A primeira super-classe demonstrada é a Planejamento e Preparacao. Essa super-classe é composta de outras 6 classes, que por sua vez, são compostas por 23 sub-classes. As sub-classes Conhecimento do conteudo e pedagogia, Conhecimento dos recursos, Conhecimento sobre os estudantes, Determinar resultados da instrucao, Preparar avaliacoes para estudantes e Preparar instrucao coerente compõem o conceito da atividade de planejamento e preparação.

Por meio de alguns indicadores levantados na nossa pesquisa, pretendemos coletar a percepção do estudante e oferecer o feedback ao professor. Para oferecer esse feedback, foram relacionados esses seguintes indicadores com a super-classe Planejamento e Preparacao: Planos de aula que reflitam conceitos importantes da disciplina, relacionamentos entre prérequisitos e conceitos/habilidades, explicações claras e precisa na sala de aula, resposta às questões dos estudantes, interesses dos estudantes e necessidades de aprendizagem, resultados que reflitam o aprendizado em diversos tipos, uso de vários materiais e recursos, entre outros.

Figura 1: A super-classe Planejamento e Preparação e suas sub-classes. 


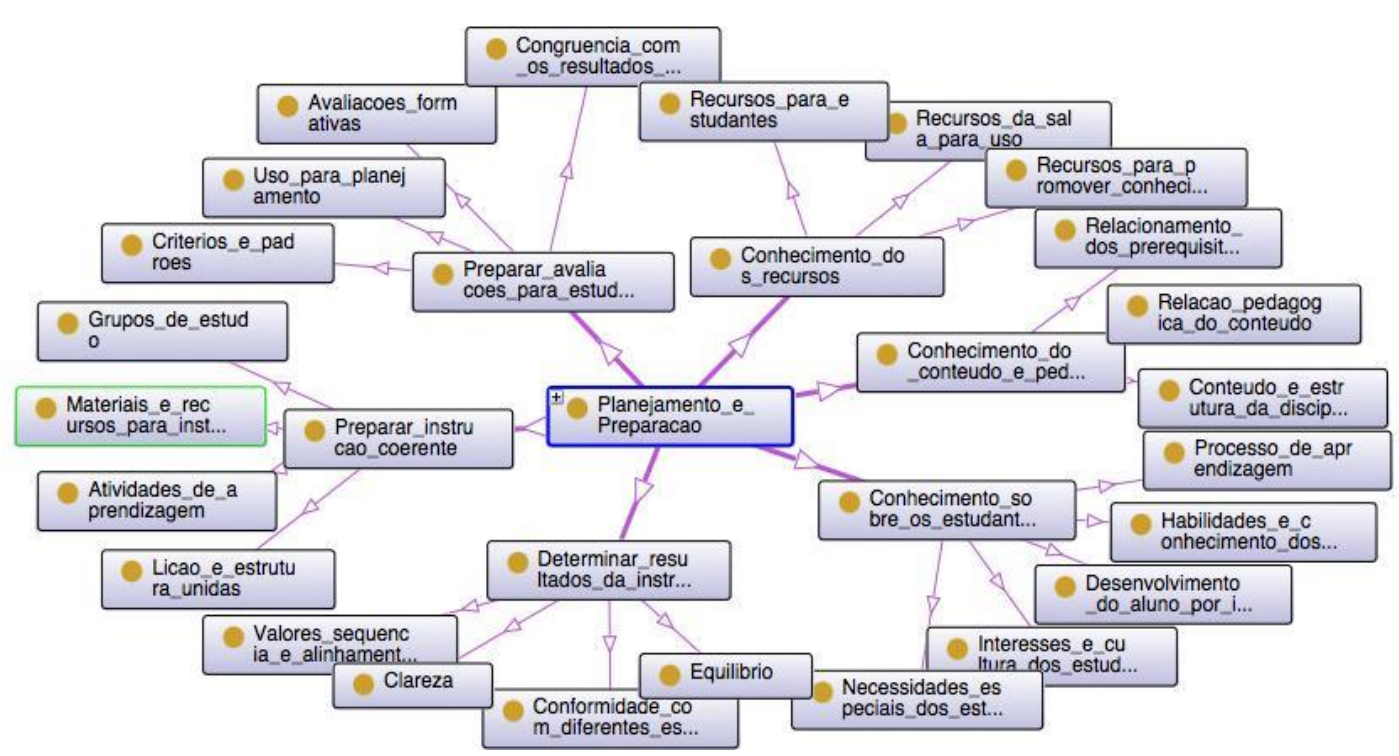

Fonte: Elaboração própria (2015).

A segunda super-classe demonstrada é a Instrucao. Essa super-classe é composta de outras 5 classes, que por sua vez, são compostas por 18 subclasses. As sub-classes Comunicacao com os estudantes, Demonstrar flexibilidade e compreensao, Envolver estudantes na aprendizagem, Usar avaliacao na instrucao e Usar tecnicas de discussao e questionamentos compõem o conceito da atividade de instrução.

Os indicadores que terão função de oferecer o feedback foram relacionados com a super-classe Instrucao. Alguns desses indicadores são: Procedimentos específicos para as atividades da lição, questões desafiadoras cognitivamente, entusiasmo dos estudantes, atenção ao nível de entendimento do aluno, adaptação da lição, entre outros.

Figura 2: A super-classe Instrucao e suas sub-classes. 


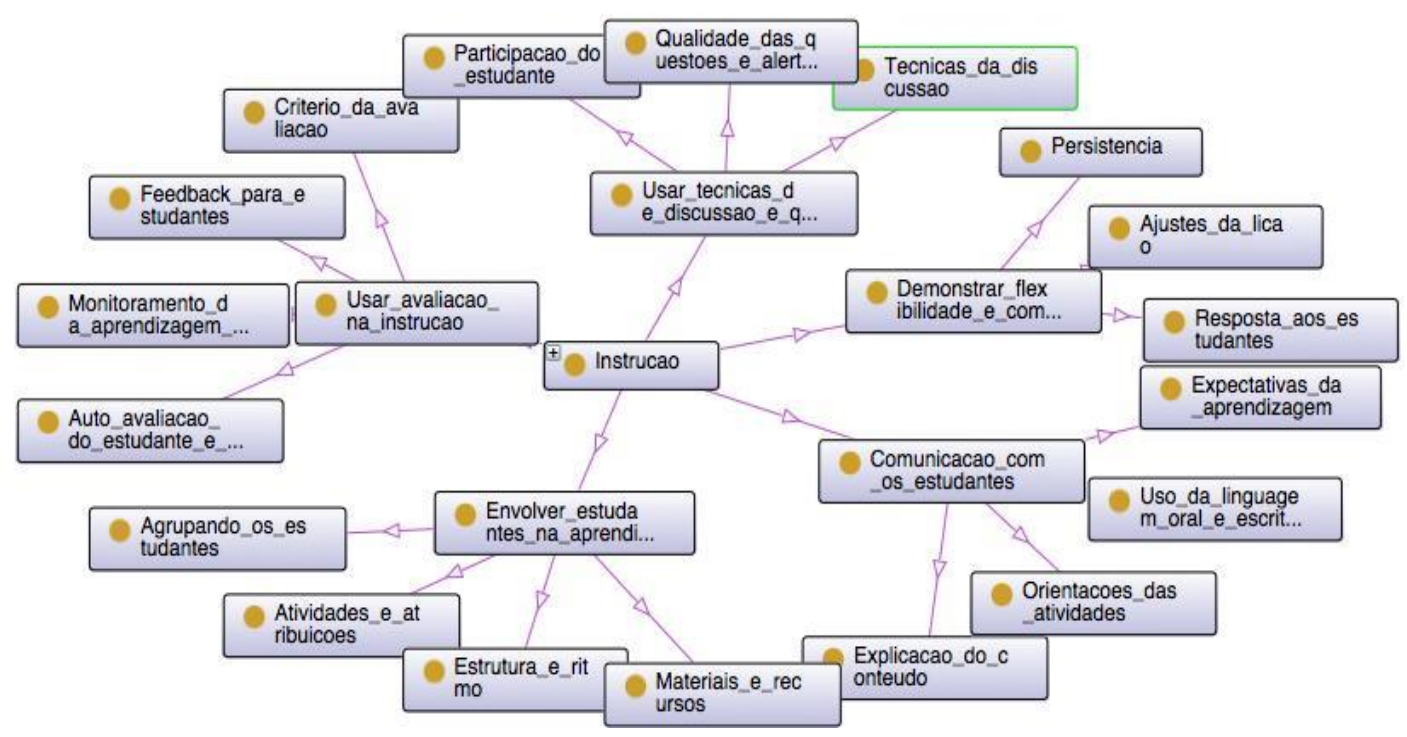

Fonte: Elaboração própria (2015).

A terceira super-classe demonstrada é a Ambiente da sala. Essa superclasse é composta de outras 5 classes, que por sua vez, são compostas por 15 sub-classes. As sub-classes Criando ambiente de respeito e empatia, Estabelecendo a cultura da aprendizagem, Gerenciando o comportamento do aluno, Gerenciando os procedimentos da sala de aula e Organizacao do espaco fisico compõem o conceito do ambiente da sala de aula.

Os indicadores que terão função de oferecer o feedback e foram relacionados com a super-classe Ambiente da sala são, por exemplo: Conversa respeitosa, escuta ativa, linguagem corporal, expectativa e reconhecimento dos esforços dos estudantes, funcionamento das rotinas, padrões claros de conduta, uso efetivo dos recursos físicos, entre outros.

A quarta super-classe que conclui a atividade de ensino é a Responsabilidades profissionais. Essa super-classe é composta de outra classe, que por sua vez, é composta por 5 sub-classes. As sub-classes Apoio, Conformidade com regulamentos da escola, Integridade e etica, Serve aos estudantes e Tomada de decisoes compõem o conceito das responsabilidades profissionais. 
Os indicadores que terão função de oferecer o feedback e foram relacionados com a super-classe Responsabilidades profissionais são: Reputação digna de confiança e muitas vezes procurado pelos alunos, age como os estudantes sejam a maior prioridade, apoia os alunos em situações políticas conflitantes, desafia práticas existentes com o foco no aluno, entre outros.

Para criação de toda a ontologia, a teoria da atividade proposta por Vygotsky (1978) foi utilizada como base. É importante salientar algumas decisões e escolhas feitas de acordo com o pensar sobre a sala de aula e prática da docência. Diante disso, alguns pontos merecem destaque para um melhor entendimento da ontologia e como serão levantados pontos específicos da docência com a percepção do aluno.

A visão de ensino interacionista e construtivista influencia nos pontos relevantes a serem considerados para oferecer o feedback. Apesar da influência dessa visão da aprendizagem no modelo, a percepção do aluno e a relevância dada ao feedback pelo professor ditará as características finais dessa arquitetura.

Para elucidação do modelo, ressaltamos alguns elementos das características da aprendizagem pensada por Vygotsky:

- A singularidade. Não existem pessoas iguais, e deve existir o respeito às diferenças individuais;

- Estimulação por meio de desafios. A tarefa do mediador é sugerir desafios, propor dificuldades e estimular competências para a superação;

- A ação mediadora de quem ensina deve visar à redução da Zona de Desenvolvimento Proximal do aprendiz. A atuação do professor sobre essa zona deve buscar o aluno interiorizar soluções e uso posterior, sem a intervenção do professor;

- A aprendizagem ocorre por assimilação de ações exteriores, interiorizadas pela linguagem que permite formar abstrações;

- Destaca as contribuições da cultura, da interação social e a dimensão histórica do desenvolvimento mental; 
- Ensinar bem não é atributo inerente a qualquer pessoa. Todos podemos ensinar, porém podemos ensinar melhor se soubermos mais sobre 0 ambiente e condições favoráveis para a aprendizagem.

\section{CONCLUSÕES}

A colaboração trazida pelo modelo deve ser apoio para que o aluno registre o máximo possível de seu conhecimento tácito, e agrupe e processe esse registro de forma efetiva junto com outros registros de conhecimentos explícitos da organização. Na construção do modelo, a utilização de uma teoria construtivista e uma abordagem multimodal agregam e enriquecem o estudo na busca por resultados e entendimento do problema. Assim que registrado o feedback por meio do modelo, através da validação dos agentes envolvidos será possível descrever e representar parte do processo que ocorre na sala de aula, trazendo novos rumos para essa área de pesquisa. A ontologia criada possibilita o seu uso em arquiteturas para geração automática de feedbacks, devido à sua formalização e amplitude semântica.

Em resumo, do ponto de vista teórico, o presente estudo traz contribuição significativa para a ciência da informação, considerando que introduz uma nova abordagem, oriunda da psicologia e da educação. Mais do que isso, acrescenta aos estudos sobre organização do conhecimento a modelagem das atividades do professor numa perspectiva aprofundada e 0 propicia o desenvolvimento de uma arquitetura da informação para gerar feedbacks nesse contexto. Do ponto de vista prático, vislumbra-se a possibilidade da aplicação de seus resultados no auxílio e capacitação dos professores, provendo um meio para o uso da informação no enfrentamento dos desafios da sala de aula e qualificação do ensino.

\section{REFERÊNCIAS}

ANTUNES, C. Piaget, Vygotsky, Paulo Freire e Maria Montessori em minha sala de aula. São Paulo (SP): Principis, 2008. 
CORCHO, O; GOMEZ-PEREZ, A. A roadmap to ontology specification languages. In: INTERNATIONAL CONFERENCE ON KNOWLEDGE ENGINEERING AND KNOWLEDGE MANAGEMENT METHODS, MODELS AND TOOLS, 12., 2000, France. Anais...Proceedings. France, 2000. p.80-97.

DALE, E. Audiovisual methods in teaching. New York: Dryden Press, 1969.

DEAN, M. et al. A. Web ontology language (OWL) reference version 1.0. W3C Working Draft, nov.2002. Disponível em:<http://www.w3.org/TR/2002/WD-owlref-20021112/>. Acesso em 19 mai 2015.

DIAS, M. L. A esfera multimodal: o uso de power point como ferramenta de expressão e integração em um ambiente educacional. 2011. Tese (Doutorado em Letras) - PONTIFÍCIA UNIVERSIDADE CATÓLICA DO RIO DE JANEIRO, Rio de Janeiro.

ENGESTRÖM, Y. Learning by expanding: an activity-theoretical approach to development research. Helsinki, Finland: Orienta-Konsultit, 1987. Disponível em: <http://lchc.ucsd.edu/MCA/Paper/Engestrom/expanding/toc.htm>. Acesso em: 18. Mai. 2015.

FEITOSA, A. A integração entre sistemas legislativos, terminologia e web semântica na organização e representação da informação legislativa. 2005. Tese (Doutorado em Ciência da Informação) - Departamento de Ciência da Informação e Documentação, Universidade de Brasília, Brasília.

FRAGOULIS, I.; DIAMANTAKI, E. The Importance of Feedback in Relation to Doing Practical Teaching Exercises. Opinions Postgraduate Student School of Pedagogical and Technological Education Heraklio of Crete. IES, v. 5, n. 6, 2012. Disponível em:

$<$ http://www.ccsenet.org/journal/index.php/ies/article/view/20858>. Acesso em 21 mai. 2015.

GRUBER, T. What is an Ontology? Disponível em: <http://wwwksl.stanford.edu/kst/what-is-an-ontology.html> 1996. Acesso em maio de 2015.

GUARINO, N; GIARETTA, P. Ontologies and knowledge bases: Towards a terminological clarification. In: N. Mars (Ed.) Towards Very Large Knowledge Bases, Knowledge Building and Knowledge Sharing. Amsterdam: IOS Press, 1995.

GUARINO, N.; OBERLE, D.; STAAB, S. What is an Ontology? S. Staab; R. Studer (eds.), Handbook on Ontologies, Second Edition. International handbooks on information systems. Berlin: Springer Verlag, 2009. p. 1-17

KRESS, G. Literacy in the new media age. London: Routledge, 2003. 
KRESS, G.; VAN LEEUWEN, T. Reading images: the grammar of visual design. New York: Routledge, 1996.

KRESS, G.; VAN LEEUWEN, T. Multimodal Discourse: the modes and media of contemporary communication. London: Arnold, 2001.

KUUTTI, K. Activity Theory as a potential framework for human-computer interaction research. In: B. Nardi, (Ed.), Context and consciouness: Activity theory and human-computer interaction. Cambridge, MA: MIT Press, 1996. p. $17-44$

LE COADIC, YF. A Ciência da Informação. Brasília: Briquet de Lemos, 2004.

Leont'ev, A. N. Activity, consciousness, and personality (M. J. Hall, Trans.). Englewood Cliffs, NJ: Prentice Hall, 1978. Disponível em: $<$ www.marx- ists.org/archive/leontev/works/1978/index.htm>. Acesso em: 18 mai 2015.

LERNER, R. Concepts and theories of human development. Mahwah, N.J.: L. Erlbaum, 2002.

LIZZIO, A.; WILSON, K. Feedback on assessment: students' perceptions of quality and effectiveness. Assessment \& Evaluation in Higher Education, v. 33, n. 3, p. 263-275, 2008.

MACHIN, D. Introduction to Multimodal Analysis. London: Hodder Arnold, 2007.

NICOL, D.; MACFARLANE-DICK, D. Formative assessment and self-regulated learning: a model and seven principles of good feedback practice. Studies in Higher Education, v. 31, n. 2, p. 199-218, 2006.

NORRIS, S. Analyzing multimodal interaction: a methodological framework. London \& New York: Routledge, 2004.

NOY, F. N.; MCGUINNESS, D. L. Ontology development 101: a guide to create your first ontology, 2001. Disponível em:

$<$ http://ksl.stanford.edu/people/dlm/papers/ontology-tutorial-noymcguinness.doc>. Acesso em: 19 maio 2015.

ORSMOND, $\mathrm{P}$ et. al. Moving feedback forward: theory to practice. Assessment \& Evaluation in Higher Education, v. 38, n. 2, p. 240-252, 2013.

PAIVA, V. L. M. O. Feedback em Ambiente Virtual. In: LEFFA, V. (Org.) Interação na aprendizagem das línguas. Pelotas: EDUCAT, 2003. Disponível em: <http://www.veramenezes.com/feedback.htm>. Acesso em: 21 maio 2015. 
PASTORE, R. Principles of Teaching. Bloomsburg, PA: Bloomsburg University, 2003. Disponível em:<http://teacherworld.com/potdale.html>. Acesso em: 19 maio 2015.

RINVOLUCRI, M. Key concepts in ELT. ELT Journal, v. 48, n. 3, p. 287-288, 1994.

ROBINSON, S.; POPE, D.; HOLYOAK, L. Can we meet their expectations? Experiences and perceptions of feedback in first year undergraduate students. Assessment \& Evaluation in Higher Education, v. 38, n. 3, p. 260272, 2013.

ROYCE, T. Multimodality in the TESOL classroom: exploring visual-verbal synergy. TESOL Quarterly, V. 36, N. 2, p. 191-205, 2002.

SANTOS, G. L. Uma Pesquisa Longitudinal sobre Professores e Computadores. Educação \& Realidade, Porto Alegre, v. 36, n. 3, p. 837-848, set./dez. 2011. Disponível em: <http://www.ufrgs.br/edu realidade>. Acesso em: 21 maio 2015.

SMITH, M, K.; WELTY, C.; MCGUINNESS, D, L. OWL Web Ontology Language Guide. Disponível em: <http://www.w3.org/TR/owl-guide/>. Acesso em: 19 maio 2015.

TANG, J.; HARRISON, C. Investigating university tutor perceptions of assessment feedback: three types of tutor beliefs. Assessment \& Evaluation in Higher Education, v. 36, n. 5, p. 583-604, 2011.

TOLMAN, C. W. Society versus context in individual development: Does theory make a difference? In: ENGESTRÖM,Y; MIETTINEN, R.; PUNAMÄKI. R. L. (Eds.), Perspectives on activity theory. Cambridge, UK: Cambridge University Press, 1999.

UR, P. A course in language teaching. Tradução nossa. Cambridge [England]: Cambridge University Press, 1996.

VIEIRA, S. A. O livro $r$ da Metafisica de Aristóteles: ontologia*- a ciência do Ser enquanto Ser. Principios, Natal, v. 2, n. 3, Jul./Dez. 1995. Disponível em: $<$ http://periodicos.ufrn.br/principios/article/view/757>. Acesso em: 18 Mai. 2015.

VYGOTSKY, L. S. Mind in society: The development of higher psychological processes. Cambridge, MA: Harvard University Press, 1978.

WILLIAMS, R. Tell me how I'm doing. New York: AMACOM, American Management Association, 2005. 


\title{
INFORMATION SYSTEM MODEL TO GENERATE FEEDBACK IN TEACHING BASED ON ACTIVITY THEORY
}

\begin{abstract}
:
Introduction: Feedback is information about a person's performance on a particular task. The lack of this item in the classroom can bring several damages to the quality of teaching. Objective: The study seeks to analyze the student's perception to generate relevant information to the teacher as feedback. Methodology: For this, we propose an innovative modeling with aspects of activity theory, multimodality and ontology use. Results: as a result, we intend to create ontology of classrooms that determine the activities of the teacher that can be aided with the students' perception in education. Conclusions: the possibility of applying their results for help and training the teachers, providing a way for the use of information in addressing the challenges of the classroom and qualification of teaching.
\end{abstract}

Descriptors: Information systems; Feedback; Teaching; Activity theory; Multimodality; Ontologies

\section{MODELO DE SISTEMA DE INFORMACIÓN PARA GENERAR FEEDBACK EN LA ENSEÑANZA BASADO EN LA TEORÍA DE LA ACTIVIDAD}

\begin{abstract}
RESUMEN:
Introducción: la retroalimentación es la información sobre el rendimiento de una persona en una tarea determinada. La falta de este ítem en el aula puede traer diversos perjuicios para la calidad de la enseñanza. Objetivo: el estudio busca analizar la percepción del alumno para generar información relevante al profesor en forma de feedback. Metodología: para tanto, proponemos un modelado innovador con aspectos de la teoría de la actividad, multimodalidad y uso de ontología. Resultados: como resultado, se pretende crear una ontología de las aulas que determine las actividades del profesor que pueden ser auxiliadas con la percepción de los alumnos en la educación. Conclusiones: la posibilidad de aplicar sus resultados para ayudar y capacitar a los profesores, proporcionando una forma de utilizar la información para abordar los desafíos del aula y la calificación de la enseñanza.
\end{abstract}

Descriptores: Sistemas de información; Feedback; Enseñanza; Teoría de la actividad; Multimodalidad; Ontología 